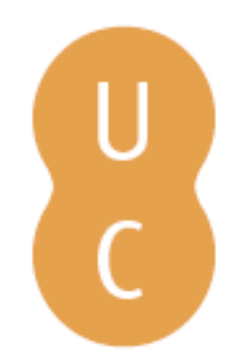

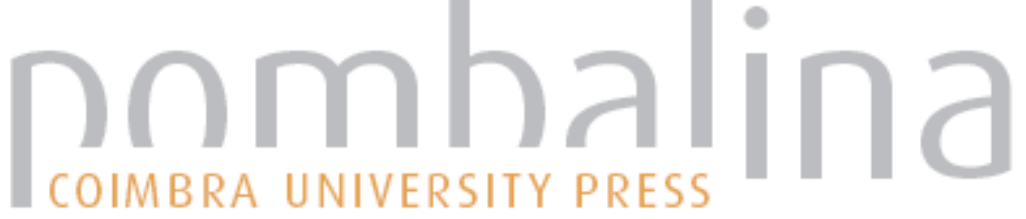

\section{Bewusster Selbstbezug ohne Regress}

Autor(es): Kübler, Stefan

Publicado por: Imprensa da Universidade de Coimbra

URL

persistente: $\quad$ URI:http://hdl.handle.net/10316.2/31631

DOI: $\quad$ DOI:http://dx.doi.org/10.14195/978-989-26-0205-9_10

Accessed : $\quad$ 26-Apr-2023 16:17:15

A navegação consulta e descarregamento dos títulos inseridos nas Bibliotecas Digitais UC Digitalis, UC Pombalina e UC Impactum, pressupõem a aceitação plena e sem reservas dos Termos e Condições de Uso destas Bibliotecas Digitais, disponíveis em https://digitalis.uc.pt/pt-pt/termos.

Conforme exposto nos referidos Termos e Condições de Uso, o descarregamento de títulos de acesso restrito requer uma licença válida de autorização devendo o utilizador aceder ao(s) documento(s) a partir de um endereço de IP da instituição detentora da supramencionada licença.

Ao utilizador é apenas permitido o descarregamento para uso pessoal, pelo que o emprego do(s) título(s) descarregado(s) para outro fim, designadamente comercial, carece de autorização do respetivo autor ou editor da obra.

Na medida em que todas as obras da UC Digitalis se encontram protegidas pelo Código do Direito de Autor e Direitos Conexos e demais legislação aplicável, toda a cópia, parcial ou total, deste documento, nos casos em que é legalmente admitida, deverá conter ou fazer-se acompanhar por este aviso.

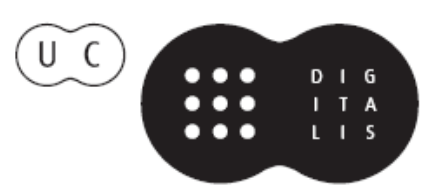


Edmundo Balsemão Pires

Burkhard Nonnenmacher

Stefan Büttner-von Stülpnagel

Editors

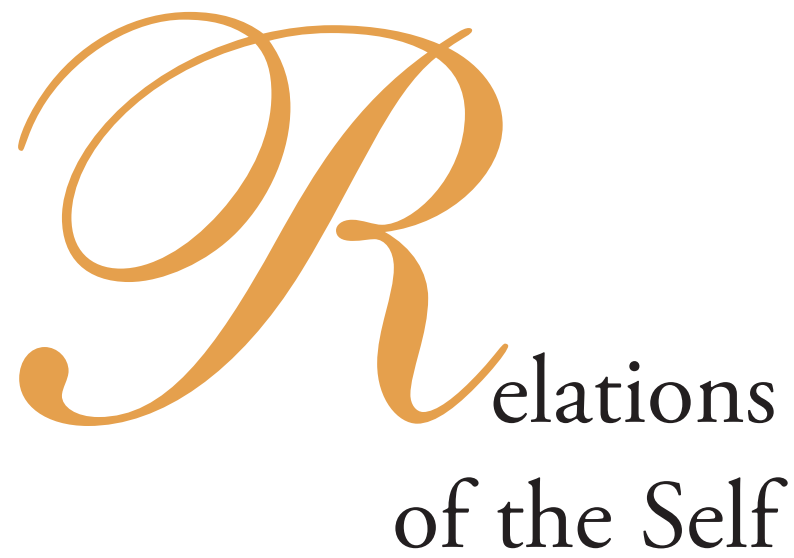




\section{Stefan Kübler \\ Tübingen}

\section{BEWUSSTER SELBSTBEZUG OHNE REGRESS}

Obwohl Hume seinerzeit dafür sprach, dass man es anders sehen solle, glaube ich an die Richtigkeit eines empirischen Befunds: Ich kann nicht nur einen Gedanken haben, sondern auch mich selbst als den Denker eines Gedankens wahrnehmen. Um diesen Befund gegen Hume aufrecht zu erhalten, wird man sicherlich an einer Stelle etwas zur grundlegenden Einheit der einzelnen Wahrnehmungen dieses Ichs sagen müssen, die er bestritt. Das werde ich hier allerdings nicht direkt tun. Stattdessen geht es mir um ein anderes Problem. Die Ich-Wahrnehmung ist ja nicht eine Objektwahrnehmung wie jede andere. Wenn das Ich sich wahrnehmen soll, muss es auf sich selbst Bezug nehmen. Es ist schon kompliziert genug, zu erklären, wie ihm das gelingt. Schlimmer ist, dass es den Anschein hat, als könne ein Ich gar nicht auftreten vor der Reflexion auf sich selbst; als würde ein Ich erst entstehen durch das Bewusstsein von sich.

Sollte das stimmen, dann setzte sich die Verteidigung einer Möglichkeit zur Wahrnehmung des Ichs dem Vorwurf aus, etwas präsentieren zu wollen, das immer schon da sein muss, bevor es existiert. Es entsteht in diesem Fall ein intensiver Regress, den man ganz kurz so darstellen kann: Reflexion bedeutet, dass sich etwas auf sich selbst zurück wendet. Reflexion bringt daher nie mehr Gehalt ins Spiel, als man zu Beginn schon hatte. Und im speziellen Fall: Durch die Reflexion auf einen mentalen Gehalt springt kein Ich aus der Kiste. Es gibt keine Aussicht, wie man bei dem Versuch, sich selbst beim Denken eines Gedankens zuzuschauen, etwas anderes als immer nur den alten Gehalt des Gedankens zutage fördern kann. ${ }^{1}$ Demnach hätte Hume recht: Wir finden immer nur Wahrnehmungen, nie ein Ich.

Damit die Intuition, dass man sich selbst im Denken zugänglich sei, angesichts dessen nicht als Illusion über Bord geht, muss man sich etwas einfallen lassen. Den Hinweis auf den intensiven Regress halte ich für richtig, Folgerungen daraus im Geist Humes aber für

\footnotetext{
${ }^{1}$ Ich spreche hier nur über den intensiven Regress: Bewusstsein scheint ein Resultat von Reflexion; und doch müsste man Bewusstsein immer schon haben, bevor man darauf reflektieren kann. Daneben gibt es den Vorwurf eines extensiven Regresses, der sich einstellt, wenn man die Grundannahme zugibt: ein mentaler Zustand könnte prinzipiell schon durch die Beleuchtung durch einen höherstufigen Zustand bewusst werden. Nun muss man aber erklären, warum der höherstufige Zustand bewusst sein kann. Wenn nun Beleuchtung durch einen höherstufigen Zustand notwendige Bedingung für Bewusstsein sein sollte, dann bräuchte man immer wieder einen höheren Zustand, und so würde die Kette nie enden.
} 
falsch. Aussicht auf Erfolg könnte es haben, wenn man die Rolle der Reflexion bei der Präsentation des Ichs abschwächt: ein Ich entsteht zwar wohl erst mit dem Bewusstsein; jedoch vor dem thematischen Bewusstsein von sich selbst.

\section{Zurückweisung höherstufiger Theorien des Bewusstseins}

Das Argument vom Leerlauf der Reflexion - frühe Stimmen, die es vorbrachten, kamen von Husserl und Sartre - startet nun nicht bei Analysen des thematischen Bewusstseins eines Subjekts von sich selbst, sondern eine Ebene tiefer: bei der Analyse des einfachen Bewusstseins, bei der gewöhnlichen Wahrnehmung, die nicht aktuell von einem „ich denke“ begleitet wird. Wie wird der repräsentationale Gehalt z.B. eines roten Würfels zum Gehalt eines Bewusstseinszustandes? Hier ist eine sehr kurze Skizze der gemeinsamen Grundannahme von höherstufigen Theorien des Bewusstseins, gegen die sich das Regress-Argument dann wendet:

Beginnen wir mit der Annahme, dass es bewusste und unbewusste mentale Zustände gibt. Da ist nun die Wahrnehmung eines roten Würfels. Manchmal ist es eine bewusste Wahrnehmung, manchmal nicht. Ein Kandidat für das unbewusste Wahrnehmen eines roten Würfels wäre, dass er irgendwo an der Peripherie meines Gesichtsfeldes auftaucht ohne dass ich darauf achte. Oder auch - um nicht dazu einzuladen, die Peripherie des Gesichtsfelds mit der Peripherie des Bewusstseins gleichzusetzen - kann er zentral und scharf in meinem Blickfeld verortet sein, aber ich beschäftige mich gerade gar nicht mit dem was ich sehe, sondern löse z.B. ein kompliziertes theoretisches Problem oder achte auf ein Musikstück. In solchen Fällen sieht es so aus, als sollten wir sagen, ich repräsentiere den roten Würfel in meinem Gesichtsfeld, aber ich tue es unbewusst.

Was muss nun geschehen, damit sich ein Subjekt seiner mentalen Zustände auch zusätzlich bewusst wird? Nun, es richtet seine Aufmerksamkeit auf den Gehalt dieses Zustands: und jetzt nimmt es den roten Würfel bewusst wahr.

Ein Befürworter einer höherstufigen Theorie des Bewusstseins würde den Schritt, der da getan wird, etwa so beschreiben: Zunächst ist da eine Wahrnehmung - ein mentaler Zustand, eine Repräsentation - die nicht von Bewusstsein begleitet ist. Der Gehalt dieses Zustandes ist ein roter Würfel. Dann gibt es einen weiteren mentalen Zustand: sein Gehalt ist die Wahrnehmung des roten Würfels. Während die Wahrnehmung des roten Würfels alleine noch unbewusst war, ist die Wahrnehmung der Wahrnehmung des roten Würfels nun bewusst.

Hier ist dann das angesprochene Problem, dem dieser Ansatz postwendend ausgesetzt ist: Die Wahrnehmung des roten Würfels war ja zunächst unbewusst. Nun wird der Gehalt des unbewussten Zustands eingeschlossen in einen weiteren, höherstufigen Zustand, der für sich genommen auch nicht bewusst ist - der es nicht sein kann, denn schließlich soll das Bewusstsein ja erst erklärt werden aus dem Zusammenspiel beider Zustände. Wie kann aber der zweite Zustand den ersten ins Bewusstsein heben? Er war doch bestenfalls eine Wiederholung des ursprünglichen Gehalts. ${ }^{2}$

2 „The phenomenologists [...] would be quite unconvinced by the claim that the relation between two otherwise nonconscious processes can make one of them conscious; they would find it quite unclear how a state without subjective or phenomanal qualities can be transformed into one with such qualitites, that is, into an 
Einige nehmen (mit den frühen Phänomenologen) an, dass es keinen Ausweg gibt und dass höherstufige Theorien des Bewusstseins nicht erfolgreich sein können. Die Wahrnehmung des roten Würfels erklärt sich nicht ausgehend von einem primären unbewussten Zustand; sondern der Würfel, wenn er wahrgenommen wird, ist der Erfahrung einfach gegeben. Wahrnehmungsinhalte haben keine Beleuchtung durch höherstufige Zustände nötig; sie präsentieren sich selbst. Dieser Vorschlag ist nicht unkontrovers, und er löst auch nicht alle Probleme. Das Eigentümliche an der Wahrnehmung kann sicher nicht schlicht auf Selbstpräsentation reduziert werden: Auch ein Kuckuck aus der Kuckucksuhr kann sich selbst präsentieren, und er ist darum doch kein Bewusstseinszustand. Dennoch möchte ich hier so viel zugestehen: nehmen wir an, eine Erklärung der Struktur der Wahrnehmung komme nicht darum herum, Selbstpräsentation mentaler Zustände zuzugestehen - was immer sich darüber hinaus sonst noch sagen lasse und sagen lassen muss. Man kann dieses Zugeständnis machen, um sich einen Standpunkt zu verschaffen, von dem aus die weiteren Probleme angegangen werden können. Denn selbst wenn wir Selbstpräsentation zugeben bleibt für das Bewusstsein eines Subjekts von sich selbst noch genug zu erklären übrig.

Wie kommen wir also von dem sich selbst präsentierenden Gehalt zum thematischen Bewusstsein von einem Subjekt? Man könnte an dieser Stelle dann doch liebäugeln mit einem höherstufigen Modell: Das Subjekt distanziert sich von der erlebten ursprünglichen Wahrnehmung und bringt so das Bewusstsein eines Ichs hervor.

Wer aber nun bezüglich der einfachen Wahrnehmung - des Gewahrseins eines roten Würfels - ein höherstufiges Modell abgelehnt hat, kann nicht ein ebensolches Modell für das Ich-Bewusstsein bemühen.

Denn geben wir zu, das Gewahrsein des roten Würfels sei gegen den Regress abgesichert durch den Umstand, dass der mentale Zustand sich selbst präsentiert. Wie nun, wenn wir wissen wollen, wie es zu der Beobachtung kommen kann, dass ich einen roten Würfel wahrnehme? Können wir ein Ich finden, wenn wir uns auf die Wahrnehmung eines roten Würfels konzentrieren? Nein: Wie sollten wir es auch in diesem Fall anstellen, um immer denselben unveränderten mentalen Gehalt herumzukommen - den guten alten roten Würfel?

Wenn das höherstufige Modell unfähig war, unsere Wahrnehmung (unser Gewahrsein) eines roten Würfels zu erklären, dann fährt es nicht besser mit der Wahrnehmung einer Wahrnehmung.

Das wird sich nicht glücklicher fügen, wenn wir nun einfach auch dem Ich erlauben, sich selbst zu präsentieren. Das Auftreten des Ichs wäre in diesem Fall nicht das Resultat einer Reflexion, es würde auf ganz eigenen Füßen stehen, unabhängig von einer einfacheren Objektwahrnehmung. Das mag eine vertretbare Überzeugung sein, aber das alleine hilft für das aktuelle Problem nicht viel. Zwar scheint absehbar: wenn sich das Ich wahrnehmen soll, dann muss es sich auf irgendeine Art selbst präsentieren. Andernfalls folgt der bekannte Regress.

Wie soll nun aber der Schritt gelingen von der Selbstpräsentation des mentalen Gehaltes (von einem roten Würfel) zur Zuschreibung des Gehalts an ein Subjekt? Doch nicht, indem sich nun obendrein das Ich selbst präsentiert. So bekommen wir kein Verhältnis zwischen der Selbstpräsentation des Ichs und der des roten Würfels zustande. Besser wäre es, wir könnten einen Weg finden, wie wir erklären, dass sich das Ich doch an der Wahrnehmung bemerkt; allerdings nicht in einer Reflexion auf sie.

experience with first personal givenness or mineness, by the mere relational addition of a meta-state having the firstorder state as its intentional object." (Dan Zahavi, Subjectivity and Selfhood, Cambridge 2008, S. 25) 


\section{Minimale Willensakte als notwendige Bedingung für Bewusstsein}

Das Problem des Regresses kommt also dann auf, wenn wir von der Auffassung ausgehen, das Ich präsentiere sich in einem Akt der Reflexion. Nun sind aber glücklicherweise die Aussichten gar nicht so schlecht, es bereits vorher zu finden. In der Tat, meine ich, finden wir es bereits in der einfachen Wahrnehmung.

Ich-los ist vielleicht der mentale Zustand bevor der rote Würfel ein Gegenstand des klaren Bewusstseins wird - wie das etwa für Dinge an der Peripherie des Gesichtsfeldes (aber nicht nur für sie) oft zutrifft. Allerdings ist es zweifelhaft, ob wir solche Fälle überhaupt als Beispiele für Bewusstsein werten sollten. Wir brauchen uns an dieser Stelle jedenfalls nicht mit ihnen zu beschäftigen, denn wer sagt, Wahrnehmungsinhalte präsentierten sich selbst und ohne Beleuchtung durch ein Subjekt, der ist ohnehin anspruchsvoller: er meint nicht die in ihrem Status zweifelhaften Gehalte, die ohne Aufmerksamkeit vorübergehen, sondern er legt sich fest auf eben die, die sich dem Bewusstsein deutlich präsentieren. Er meint alle die Gehalte, die nicht von einem ,ich denke“ aktuell begleitet sind; unter ihnen sind aber eine ganze Reihe, die klar dem bewussten Erleben zuzuschlagen sind.

Und alle diese sind bereits nicht mehr anonym. Jeder klare Bewusstseinszustand gehört auch einem Subjekt an, das ihn sich zuschreibt - wenngleich nicht thematisch, sondern auf eine undistanzierte praktische Weise, aber doch eine Weise, die innerhalb des Bewusstseins und der Perspektive der ersten Person stattfindet. Das bestätigt sich, wie wir gleich sehen werden, an unserem Umgang mit solchen Wahrnehmungen.

Hier ist der Angelpunkt für die praktische Zuschreibung: Jede Wahrnehmung wird von einem mindestens minimalen Willensakt begleitet. Was reine phänomenale Gehalte an ein Subjekt binden könnte, wäre tatsächlich rätselhaft. Wie Willensakte binden ist dagegen schon anschaulicher.

Bevor ich weiter gehe, möchte ich eine etwas lose „Definition“ dessen voranstellen, was ich mit einem (minimalen) Willensakt meine:

Als (minimalen) Willensakt verstehe ich eine Bereitschaft zu Handeln, die phänomenaler Teil des Bewusstseinszustands ist. Eine phänomenale Qualität hat sie als (affektive) Bewertung des eigenen Zustands im Verhältnis zum Objekt. Dabei muss diese Bereitschaft aber nicht thematischer Teil des Bewusstseinszustands sein: Es genügt, dass sie - aufgrund der Rolle, die sie in diesem Bewusstseinszustand aktuell spielt - jederzeit zum Thema werden kann (eine Disposition dazu, unmittelbar thematisch zu werden, wenn die Umstände entsprechend sind).

Diese Fassung von „Willensakt“ ist ziemlich weit. ${ }^{3}$ Allerlei fällt darunter, was man sonst oft gerne vom Willen absetzt: Begierden, wenigstens einige Emotionen; und darf man in solchen Phänomenen wie dem beinahe teilnahmslosen Beobachten der ziehenden Wolken noch einen Willensakt suchen? Die Rechtfertigung liegt darin: Ich ziele auf die Behauptung ab, dass jedes Bewusstsein mit einer (minimalen) Bereitschaft zu Handeln verbunden ist. Entscheidend ist dabei, dass es sich um kausale Fähigkeiten innerhalb der phänomenalen

\footnotetext{
${ }^{3}$ Sie ist damit Frankfurts „Willenakten erster Stufe“ sehr ähnlich: Harry Frankfurt, „Freedom of the Will and the Concept of a Person“, in ders. , The Importance of What We Care About, Cambridge 2005.
} 
Perspektive handelt. Ein Willenakt ist der typische Fall von mentaler Verursachung, und so liegt es nahe, darin den Oberbegriff für alle mentale Verursachung zu erkennen - jedenfalls für alle, die als Motivation wirkt und so auf Handlung ausgerichtet ist. ${ }^{4}$

Dieser (minimale) Willensakt ist Teil der phänomenalen Qualität des Bewusstseinszustandes, auch wenn er gerade nicht Thema des Bewusstseins ist.

Vergleiche:

„I believe that the avoidance of boredom is a very fundamental human urge. It is not a matter merely of distaste for a rather unpleasant state of consciousness. Being bored entails a reduction of attention; our responsiveness to conscious stimuli flattens out and shrinks; distinctions are not noticed and not made, so that the conscious field becomes increasingly homogenous. The general functioning of the mind diminishes. It is the essence of boredom that it involves an attenuation of psychic liveliness. Its tendency is to approach a complete cessation of significant differentiation within consciousness; and this homogenization is, at the limit, tantamount to the cessation of conscious experience altogether." 5

Frankfurt spricht von Langeweile, aber von Langeweile in einem so vollkommenen Sinn, dass damit nur noch etwas unserer gewöhnlichen Langeweile Analoges gemeint sein kann: Wer im gewöhnlichen Sinn gelangweilt ist, findet sich in einem unangenehmen Zustand. Soweit es geht, wird er versuchen, ihn zu vermeiden, und sei es nur, indem er von einem langweiligen Fernsehprogramm zum nächsten schaltet, um wenigstens etwas zu tun, während er die Hoffnung aufgegeben hat, überhaupt etwas Besseres zu finden. Vielleicht tut er auch tatsächlich gar nichts mehr, aber würde doch wohl gerne, wenn er nur wüsste, was. Das ist schlimm genug, aber die totale Langeweile, besser: das absolute Desinteresse, ist nicht schlimm, oder fühlt sich jedenfalls nicht so an: es ist gar nicht zu bemerken. Denn es schließt natürlich das Desinteresse an dem Zustand, in dem man sich befindet, ein.

Obwohl ich also mit Frankfurt einig bin, dass ein Zustand, in dem der Wille vollständig betäubt ist, kein bewusster Zustand sein kann, möchte ich sein Beispiel doch eher dazu hernehmen um anschaulich zu machen, in welchen Fällen man - vielleicht überraschenderweise - immer noch einen aktiven Willen beobachten kann. Langeweile ist ein solcher Fall. An ihr kann ich sogar gut verdeutlichen, was ich mit phänomenaler Qualität der Willenseinstellung meine. Langeweile ist bemerkbar, und sie hat eine phänomenale Qualität. Ihre phänomenale Qualität hat sie von der Willenseinstellung her, die sie sogar konstituiert. Obwohl es schwer ist, zu sagen, was für eine Willenseinstellung genau Langeweile konstituiert, wird man sagen können, dass es u.a. ein Bedürfnis nach Tätigkeit ist.

\section{Reine phänomenale Zustände - anonyme Erfahrung?}

Versuchen wir die Gegenprobe. Finden wir Wahrnehmungen ohne minimalen Willensakt, die dennoch bewusst sind? Hier wären Kandidaten dafür: Schmetterlinge haben Augen, und

\footnotetext{
${ }^{4}$ Denn man kann vielleicht auch dafür sprechen, dass z.B. in der Erinnerung noch eine andere Weise mentaler Verursachung stattfinde. Etwa: „Ein Sinneseindruck verursacht eine Erinnerung.“

${ }^{5}$ Harry Frankfurt, „On the Usefulness of Final Ends”, in: Ders, Necessity, Freedom, and Love, Cambridge 1999 , S. 89.
} 
sie können möglicherweise Farben unterscheiden; Schnecken können vielleicht riechen. Nehmen wir an, Schnecken und Schmetterlinge verfügen nicht über minimale Willensakte. Zusammen mit der Forderung, dass minimale Willensakte notwendige Bedingung für Bewusstsein seien, bekommen wir ein Resultat, das fasst paradox erscheinen wird: Schmetterlinge und Schnecken verfügen über phänomenale Zustände, sie haben aber kein Bewusstsein. Dieses Resultat möchte ich im Zweifelsfall verteidigen. Man könnte das angreifen, indem man darauf verweist, dass „phänomenal“ immer auch schon „bewusst“ impliziert. Das ist eine weit verbreitete Überzeugung, sie stellt uns aber vor Probleme, wenn wir erklären wollen, wie die Sinneseindrücke von Schnecken und Schmetterlingen mit unseren der Art nach kontinuierlich sein können. Gleiches gilt für mentale Zustände im menschlichen Geist, die völlig unbemerkt vorübergehen. Diese und die Repräsentationen von Schnecken und Schmetterlingen mögen wohl immer noch etwas anderes sein als die Repräsentation der Temperatur durch einen Thermostaten: Sie sind näher am Bewusstsein, aber dennoch kein Bewusstsein. Es ist eine Möglichkeit, diese Nähe auszudrücken, wenn man von phänomenalen Zuständen spricht, die nicht bewusst sind.

Ist das aber nicht widersprüchlich? Sind phänomenale Zustände nicht deswegen schon bewusst, weil sie sich „irgendwie anfühlen“? Fühlen sich denn reine phänomenale Zustände irgendwie an? Wenn in der Tat ein phänomenaler Zustand ein solcher Zustand ist, der sich „anfühlt“, dann fühlt er sich in der Regel für ein Subjekt irgendwie an. Dieses „SichAnfühlen“ hat aber wohl zwei Komponenten: einerseits gibt es ein „Sich-Anfühlen“ des Farbeindrucks, und damit von dieser Seite her eines Gehalts, der nicht vollständig durch eine Beschreibung darzustellen ist, sondern nur so, dass man zusätzlich auf eine sinnliche Qualität hinweist. Zweitens fühlt es sich auch irgendwie an, als Subjekt eine bestimmte Position dieser Qualität gegenüber zu haben, und auch das ist ein auf objektive Beschreibung nicht reduzierbares Ereignis. Farbeindruck und phänomenale Repräsentationen der eigenen Position fühlen sich für gewöhnlich gemeinsam irgendwie an. Beide Seiten haben Elemente, die nicht reduzierbar sind auf objektive Beschreibungen, sondern angefühlt werden müssen, um gehabt zu werden. Wenn von diesen Elementen aber eine Sorte die Chance haben sollte, sich alleine irgendwie anzufühlen, dann ist es nur die Erfahrung der eigenen Position. In (minimalen) Willensakten ist das Subjekt sich immer selbst mitgegeben; in reinen Farbeindrücken wäre nur die Außenwelt gegeben.

Es scheint im Prinzip nichts dagegen zu sprechen, Schnecken und Schmetterlingen ein voll ausgeprägtes phänomenales Wahrnehmungsvermögen für Gerüche und Farben zuzuschreiben. Hätten sie aber deswegen wirklich Bewusstsein? Ich neige dazu, es ihnen abzusprechen: sie sind völlig bewusstlos, und sie sind es insbesondere auch in Bezug auf Farben und Gerüche.

Nun will ich allerdings gar nicht soweit gehen, phänomenale Zustände, die nicht bewusst sind, offensiv zu behaupten. Wenn wir nun etwas über den Farbeindruck sagen wollen und dabei die Position, die das Subjekt ihm gegenüber hat, völlig außen vor lassen wollen - sollen wir diesen Farbeindruck dann einen phänomenalen Zustand nennen oder nicht? Vielleicht ist ein phänomenaler Zustand ohne Bewusstsein Unsinn - dann sind reine Farbeindrücke theoretische Konstrukte und keine phänomenalen Zustände. ${ }^{6}$ Vielleicht ist er

${ }^{6}$ Das wäre dann der Fall, wenn sich nachweisen ließe, dass Farbeindruck und der sich auf ihn richtende minimale Willensakt letztendlich gar nicht ganz voneinander verschieden sind. Aber dieser Nachweis wird wohl kaum sicher zu führen sein. 
kein Unsinn - dann sind reine Farbeindrücke zwar phänomenale Zustände, aber in völliger Bewusstlosigkeit.

Welche der beiden Alternativen zutrifft ist nicht entscheidend. Wichtig ist aber, was phänomenale Zustände wären, wenn sie ganz abgekoppelt vom Handeln vorkommen könnten - oder, mit anderen Worten, vorkommen könnten ohne einen (minimalen) Willensakt, der sich auf sie bezieht: Sie wären genau die Zustände, die Schmetterlinge und Schnecken haben können. Das heißt mit hoher Wahrscheinlichkeit: sie wären keine bewussten Zustände. Wichtig ist, dass es keine bloßen phänomenalen Zustände geben kann, die allein dadurch, dass sie phänomenalen Gehalt haben, auch tatsächlich bewusst sind. Auch einfache Wahrnehmungen sind demnach nicht zunächst anonym, um erst in der Reflexion von einem Subjekt angeeignet zu werden.

Um meinen Einsatz für diese These zu verstärken, will ich nun versuchen, die andere Seite darzustellen: Wir hatten klare Fälle von anonymen mentalen Zuständen, die sich als nicht bewusst herausstellten. Betrachten wir nun klare Fälle von Bewusstsein, die nicht als anonyme - Subjekt- oder Ich-lose - Bewussteinszustände gelten dürfen; obwohl die Reflexion des Subjekts auf sie sehr weit zurück genommen ist.

Martin beschreibt solche Zustände mehr oder weniger alltäglicher Selbst-loser Erfahrung, um mit ihnen an die esoterische Selbst-lose Erfahrung („no-self experience“) heranzuführen:

„For instance, consider what your experience was like during periods of extreme concentration, such as when you were totally absorbed in working on an intellectual problem. Or consider what it was like at times when your activity was sensually overwhelming, say, during sex or swimming, or listening to music. Probably at some such times you were, as we say, totally lost in your activity."

Eine solche Erfahrung möcht er Selbst-los nennen, weil es kein „Beobachterselbst” gibt, das während eines solchen Zustands der Versunkenheit auf diesen Zustand reflektiert. Der gewählte Name - „no-self Experience“ - weist allerdings in die falsche Richtung. Es ist zwar durchaus bemerkenswert, dass man sich darüber streiten kann, ob es treffender ist, dieses Phänomen zu beschreiben als „völliges Verloren-Sein“ oder als „völliges Bei-Sich-Sein“. Hier ist aber mein knappes Argument für das Zweite: Man wird es gar nicht gerne dulden, aus der entsprechenden Aktivität herausgerissen zu werden. Das bedeutet nichts anderes, als dass man sich das Erlebnis eindeutig angeeignet hat.

Aber verfolgen wir den Vorschlag der Ich-losen Erfahrung noch etwas weiter. Parfit möchte uns eine Welt entwerfen, die von Menschen bewohnt sind, die nicht über das Konzept „Ich“ verfügen. Wie sich herausstellt, kommt ihm dabei unter der Hand eine Welt heraus, die - Parfit selbst hat diese Wendung wohl nicht beabsichtigt - von geborenen Meditationsmeistern bewohnt ist:

"In place of the pronoun "I" these beings might have a special use of "this" which referred to the sequence in which this use of "this" occurred. When one of us would say "I saw the Great Fire", one of them would say: "This included a seeing of the fire" (...) They

\footnotetext{
${ }^{7}$ Raymond Martin, Self-Concern, Cambridge 1998, S. $142 \mathrm{f}$.

${ }^{8}$ Vgl. Marya Schechtman, „Self-Expression and Self-Control“, in: Ratio 17 (2004)
} 
also might have a special use of "here", so that, instead of "I am angry" they would say "Anger has arisen here".

Nun ist ein solcher Zen-Planet zwar vielleicht denkbar. Zunächst einmal sollte aber schnell klar sein, dass die Weise, wie diese Wesen Erlebnisse haben, von unserer sehr verschieden ist. Sie ist in einem deutlich tieferen Sinn unterschieden, als Parfit zugestehen möchte:

„More exactly, since our concept of experience is the concept of an event that involves a subject, these imagined beings might not have our concept of an experience. But they might have a variant of this concept, and one that is similar enough to count as applying to the same part of reality." ${ }^{10}$

Ähnlich unserem gewöhnlichen Erleben mag der Zustand ja durchaus sein. Aber gibt das Parfit wirklich die Erlaubnis, kein Wort darüber zu verlieren, welche Mühe es uns kosten würde, diese unpersönliche Perspektive durchzuhalten - sofern es nicht eine bloße Redeweise sein soll? Wenn Winnetou zu sich „Winnetou“ sagt oder der König „Wir“, dann sind das schlechte Belege dafür, dass sie auf das Konzept „Ich“verzichten, wenn sie über ihre Erfahrungen sprechen. Wenn hinter der Redeweise eine entsprechende Einstellung stecken soll, dann ist es eine, die wir allenfalls mit viel Übung durchhalten könnten. Wir sind jedenfalls nicht die geborenen Zen-Meister. ${ }^{11}$

Aber auch Parfits Gesellen sind es nicht. Hier ist ein Beispiel:

"In the mind of our imagined mountaineer, a few connected thoughts might be as follows: ,Was it wisely decided here to make an attempt to the summit? Since a storm is coming, this may not have another chance. Should this include a crossing of that ridge of ice? The pain of the wind against this face hardly matters with a view like that.' "12

In diese Sequenz hat Parfit einige Gedanken aufgenommen, die der Bergsteiger gar nicht haben dürfte. Wie soll er wissen, ob es eine kluge Entscheidung war, den Anstieg zu versuchen? Was wäre der Maßstab dafür? Wenn diese Entscheidung ein unpersönliches Ereignis sein soll - eines, das keinen Akteur verlangt -, dann kann es darum gehen, zu beurteilen, ob es vielleicht ein Unglück war, dass sie statt gefunden hat. Es ist aber nicht angemessen, das Eintreten oder Ausbleiben von Unglücken als „weise“ zu charakterisieren.

Wie soll der Bergsteiger aber außerdem wissen, ob der Schmerz im Gesicht von Bedeutung ist? Und wie soll er die Bedeutung des Schmerzes vergleichen mit der Bedeutung der Gefahr und beides gegeneinander aufwiegen? Es ist schon denkbar, dass man in einer bestimmten Situation ein Gefühl der Distanz zwischen den eigenen Erlebnissen und sich

${ }^{9}$ Derek Parfit: „Experiences, Subjects, and Conceptual Schemes“, Philosophical Topics, 26:1/2 (1999), S. 229

${ }^{10}$ ebd., S. 228

11 Bemerkenswert ist nebenbei, dass Forman, der aus eigener Anschauung von Meditationserfahrungen berichtet, trotz einer inneren Distanz zu seiner Wahrnehmung der Außenwelt nicht nur immer noch von sich spricht, sondern sogar von seinem „wahren Selbst“, das sich in dieser Erfahrung zeigt. Vgl. Robert Forman: „What Does Mysticism Have to Teach Us About Consciousness?“ in: Ders. et al. (Hrsg.), Models of the Self, ohne Ort 2002. Wenn ich ihn richtig deute, würde er nicht sagen: „There is thinking (of xy) here“, sondern: „There is thinking within $m e^{“ .}$. Parfit muss nun nicht an Meditationspraxis denken, wenn er seine unpersönlichen Denker vorstellt. Aber wenn Meditationspraxis kein Beispiel ist für das, woran Parfit denkt - was wäre dann eins? Wenn wir trotz ernsthafter Bemühung keines finden, nährt das die Zweifel, dass man eins finden kann.

${ }^{12}$ Derek Parfit, „Experiences, Subjects, and Conceptual Schemes“, Philosophical Topics, 26:1/2 (1999), S. 229 
selbst hat. Der Bergsteiger kann sich die Ereignisse (im Extremfall) etwa so repräsentieren wie einen Film, den er betrachtet. Er kann aber nicht über die Ereignisse urteilen, wenn er $\mathrm{zu}$ allen seinen Erlebnissen immer so steht, wie der Zuschauer zum Film. Insbesondere würde er dann nicht wissen können, was es heißt, zu handeln. (Zwar nimmt Parfit Handlungen in die Liste der Ereignisse auf, die dem Bergsteiger geschehen, aber gerade dadurch, dass die Handlung ihm nur geschieht, bekommt das Wort einen hohlen Klang.)

Wenn es nun aber möglich sein sollte, dass man sich zu seinen Erlebnissen immerhin manchmal so stellen kann, wie ein Zuschauer zum Film, ist auch damit noch nicht gezeigt, dass man sich dabei gar keine Erlebnisse als Subjekt zuschreibt: Man müsste zeigen, dass man sich die Position des Zuschauers gleichfalls nicht zuschreibt. Wenn der Bergsteiger das aber nicht tut, kann er wieder nicht überlegen, ob da eine weise Entscheidung stattgefunden hat. Das Urteil, ob etwas klug getan war, verlangt den Standpunkt eines Akteurs: Dieser Akteur muss sich nicht aktuell engagieren, aber er muss aktuell klar wissen, was es heißt, sich zu engagieren.

Der Monolog des Bergsteigers liefert also kein Beispiel für eine Sprache, in der von subjektiven Zuständen im unpersönlichen Modus gesprochen wird. Kann es eine solche Sprache aber geben? Kann es denn sein, dass ein Zuschauer eine Szene als weder interessant noch langweilig, weder angenehm noch unangenehm noch irgendetwas dergleichen empfindet? Meine Überzeugung ist: wenn er sie wirklich sieht, dann muss er zugleich damit eine Bewertung abgeben, die über den eigenen Standpunkt urteilt. Das Urteil umfasst mehr als die Auszeichnung eines Erlebnisses als hier und jetzt - es muss eine (minimale) Ausrichtung auf das eigene Handeln stattfinden (erfassbar an Fragen derart: Soll die Konfrontation mit dem Erlebnis beibehalten werden oder soll man der Situation aus dem Weg gehen? Ist das gerade wichtig? etc.). Wenn das geschieht, dann ist das aber nichts anderes, als die Zuschreibung des Erlebnisses an ein Subjekt - ganz gleich, ob der Sprecher eine peinliche Übung daraus macht, von seinen Erlebnissen immer im Passiv zu sprechen: „Hier wird gesehen, gedacht, gefürchtet" etc.

\section{Wahrnehmung des Ichs}

Und wie nehmen wir das Ich nun wahr? Angenommen, es stimmt, dass ein Wahrnehmungsakt gar nicht möglich ist ohne eine engagierte Positionierung des Subjekts. Aber auch dann ist es doch ein Unterschied, diese Positionierung nur zu haben und sie obendrein zu bemerken. Oder am Beispiel: Es ist ein merklicher Unterschied zwischen der Wahrnehmung eines roten Würfels und dem thematischen Bewusstsein, dass ich einen roten Würfel wahrnehme. Der Schritt vom einen zum anderen ist nicht einfach eine Reflexion auf den Wahrnehmungsgehalt; sondern wenigstens auch eine Reflexion über die Position des Subjekts. Darauf zu reflektieren, was es heißt - oder „wie es sich anfühlt“-, dass ich einen roten Würfel beobachte, verlangt mehr, oder eigentlich: etwas ganz anderes, als die Wahrnehmung mit einer Meta-Wahrnehmung zu umwickeln. Ist es denn überhaupt im echten Sinn eine Reflexion? 
Vielleicht kommen wir noch ein Stück weiter, wenn wir uns genauer anschauen, wo das minimal willentliche Element in der Beobachtung des roten Würfels liegt. Man begegnet dem Würfel nicht mit Angst, man begehrt ihn nicht, man findet ihn weder schön noch anderweitig besonders, noch aufregend; er wird nur gesehen. Richtet man aber seine Aufmerksamkeit darauf, wie es ist, den Würfel zu betrachten, dann muss man ein Verständnis davon haben, wie es ist, etwas anderes zu tun. Fragt man sich, was das Besondere an einer Beobachtung ist, dann muss man die eigene Fähigkeit klar vor Augen haben, andere Beobachtungen zu machen oder ganz andere Dinge zu tun. Wenn es richtig ist (wie ich es glaube), dass die Reflexion auf ein Ich im Kern in einer Thematisierung der Tätigkeit des Ichs besteht, dann liegt es nahe zu vermuten, dass diese Fähigkeit eng zusammenhängt mit der Fähigkeit zu kontrafaktischem Denken. Was „weak first-person phenomena“ (mit Baker ${ }^{13}$ zu reden), von denen auch das Bewusstsein der Tiere geprägt ist, vom thematischen Nachdenken über die Tätigkeit des Ichs („strong first-person phenomena“) unterscheidet, wäre dann nicht eine gehobene Brechung der ursprünglichen Wahrnehmungsinhalte in einer Schleife, in der sich das Bewusstsein auf sich selbst richten muss. Es wäre eher ein Nachdenken über mögliche andere Umstände, denen man ausgesetzt sein könnte und - vor allem - denen man sich selbst aussetzen kann. Verfügt man über diese Fähigkeit, dann hat man, wie das Beispiel von Parfits Bergsteiger zeigt, auch damit zugleich bereits Selbstbewusstsein. Wie es sich anfühlt, in einer bestimmten Situation zu sein, vergegenwärtigt man sich dadurch, dass man sie mit anderen Situationen vergleicht. Damit erklimmt man aber nun keine höhere Bewusstseinsstufe mehr: Wenn jemand ganz in seine Lektüre versunken plötzlich in das alltägliche Leben zurück gerufen wird, besteht seine Überraschung alleine darin, Umstände wieder zu bemerken, die er vergessen hatte. Sie besteht nicht darin, ausgerechnet sich selbst zu finden und keinen anderen; ebenso wenig darin, plötzlich zu bemerken, dass er existiert.

${ }^{13}$ Lynne Rudder Baker, Persons and Bodies, Cambridge 2000. 